\title{
The PPAR $\gamma$ Agonist Rosiglitazone Impairs Colonic Inflammation in Mice with Experimental Colitis
}

\author{
JULIAN D. RAMAKERS,,${ }^{1,4}$ MARLEEN I. VERSTEGE, ${ }^{2}$ GEERTJE THUIJLS,${ }^{3}$ ANJE A. TE VELDE, ${ }^{2}$ \\ RONALD P. MENSINK, ${ }^{1}$ and JOGCHUM PLAT ${ }^{1}$
}

Received November 13, 2006; accepted January 19, 2007 Published online: 14 February 2007

\begin{abstract}
Various animal models showed that peroxisome proliferatoractivated receptor (PPAR) $\gamma$ agonists, when given as a gavage shortly preceding colitis induction, protect against inflammatory bowel disease (IBD). We have examined the effects of 16 days rosiglitazone treatment via the diet prior to dextran sodium sulphate (DSS)-induced colitis in mice. After 7 days DSS in the drinking water, rosiglitazone-fed mice had lost significantly more weight than control mice. Rosiglitazone-treated mice had more diarrhea, weight of colon and spleen were increased, and length of colon was decreased. Histology showed that rosiglitazone-treated mice had more severe colitis, mainly caused by more ulceration, crypt loss, and edema. Immunofluorescence showed a loss of tight junction structure Zonula Occludens protein 1 (ZO-1) in colons of rosiglitazone-treated mice as compared to the control mice. Also, serum amyloid $\mathrm{P}$ component (SAP) concentrations in plasma were increased. However, concentrations of tumor necrosis factor (TNF)- $\alpha$ and interferon (IFN)- $\gamma$ in colon homogenates, and TNF- $\alpha$ in spleen homogenates were significantly decreased, whereas interleukin (IL)-10 in spleen homogenates was increased. Other cytokines (IL-2, IL-4, IL-6, IL-12p70 and monocyte chemotactic protein (MCP)-1) and myeloperoxidase (MPO) concentrations showed no differences. In conclusion, 16 days pretreatment with rosiglitazone impaired DSS-induced colitis in mice.
\end{abstract}

KEY WORDS: DSS-induced colitis; rosiglitazone; peroxisome proliferator-activated receptor; PPAR.

\footnotetext{
${ }^{1}$ Department of Human Biology, Nutrition and Toxicology Research Institute Maastricht (NUTRIM), Maastricht University, P.O. Box 616, Maastricht, $6200 \mathrm{MD}$, The Netherlands.

${ }^{2}$ Department of Experimental Internal Medicine, Academic Medical Centre, University of Amsterdam, Amsterdam, The Netherlands.

${ }^{3}$ Department of Surgery, Nutrition and Toxicology Research Institute Maastricht (NUTRIM), Maastricht University, Maastricht, The Netherlands.

${ }^{4}$ To whom correspondence should be addresed; e-mail: j.ramakers@hb. unimaas.nl.
}

\section{INTRODUCTION}

Peroxisome proliferator-activated receptor (PPAR) $\gamma$ is a nuclear hormone receptor, controlling the expression of numerous genes among others that are involved in inflammation (1). PPAR $\gamma$ activation dampens the inflammatory response by decreasing NF- $\kappa$ B activation. PPAR $\gamma$ is highly expressed in the intestine, suggesting an important role in the regulation of the intestinal inflammatory response $(2,3)$. In line with this assumption, heterozygous $\operatorname{PPAR} \gamma$ deficient $(+/-)$ mice are more susceptible to experimental-induced colitis than wild-type mice $(4,5)$. In addition, treatment with $\operatorname{PPAR} \gamma$ agonists protected against colonic inflammation in different animal models of inflammatory bowel disease (IBD) (4-12). Also in humans, the PPAR $\gamma$ ligand rosiglitazone may have positive effects as suggested by the number of patients that achieved clinical and endoscopic remission in an openlabel single-arm trial for ulcerative colitis (UC) patients (13). Unfortunately, this study did not include a control group, which makes it difficult to draw any firm conclusions. Yet, these studies together do suggest that PPAR $\gamma$ activation dampens intestinal inflammation. However, in the studies concerning animals, only the effects of treatment with PPAR $\gamma$ agonists at the first day of colitis induction $(4,11,12), 1$ or 2 days before colitis induction $(4,5,7$, $8,10)$ or after colitis induction $(5,6,12)$ were examined. However, in reality, IBD patients should be treated with $\operatorname{PPAR} \gamma$ agonists such as rosiglitazone for longer periods, either when applied preventive to avoid the occurrence of relapses (as a maintenance therapy) or curative during acute exacerbations. Looking at the current literature, the latter approach, i.e., 14 days chronic rosiglitazone treatment during chronic trinitrobenzensulfonic acid (TNBS) colitis induction (6) has been evaluated, whereas the first longer term preventive approach has not been done. Thus, studies are lacking, which evaluates effects of longer term 
$\operatorname{PPAR} \gamma$ agonist treatment before colitis induction. The aim of the present study was, therefore, to examine the in vivo effects of longer term preventive treatment with the PPAR $\gamma$ ligand rosiglitazone on dextran sodium sulphate (DSS)-induced colitis.

\section{MATERIALS AND METHODS}

\section{Experimental Design}

All experiments were approved by the Animal Studies Ethics Committee of the University of Amsterdam, The Netherlands. Twenty eight-week-old female wildtype C57BL/6 mice were obtained from Charles River (Horst, The Netherlands). The mice were housed under standard conditions (AM-III 15 mm, Hope Farms, Woerden, The Netherlands) and had free access to water and food. The mice were randomly assigned to two groups, which received either control chow (control group) or control chow enriched with $12 \mathrm{mg} / 100 \mathrm{~g}$ rosiglitazone (Avandia, GlaxoSmithKline, Brentford, UK) (rosiglitazone group) for 16 days before colitis induction. Treatments were continued during the week of colitis induction. Both diets consisted of $21 \mathrm{En} \%$ protein, 66 En\% carbohydrates, and 14 En\% fats. Both diets were made by Special Diet Services (SDS, Essex, UK) and irradiated at $25 \mathrm{kGy}$. Colitis was induced by administration of $1.5 \%$ (w/v) DSS (MW $40 \mathrm{kDa}$; TdB Consultancy, Uppsala, Sweden) to the drinking water of the mice for 1 week. Body weights of the mice were recorded every third day, but were recorded daily in the week of colitis induction. After 7 days DSS treatment, all mice were anesthetized with fantanyl-fluanisone-midazolan (FFM) and sacrificed via a cardiac puncture and exsanguinations. Blood was sampled in EDTA tubes (Becton Dickinson Vacutainer Systems, Franklin Lakes, NJ) and plasma was obtained by centrifugation at $2000 \mathrm{~g}$ for $20 \mathrm{~min}$ at $4^{\circ} \mathrm{C}$ and stored at $-80^{\circ} \mathrm{C}$ until analysis for myeloperoxidase (MPO) and serum amyloid P component (SAP) concentrations. Through a midline incision, the colons were removed. First, the total length was measured as an indicator of disease-related colon shortening. In addition, fecal material was removed and collected to score diarrhea severity; 0-normal feces, 1-loose stool, 2-watery diarrhea, 3-slimy diarrhea, little blood, and 4-very severe diarrhea. Next, the colons were opened longitudinally and the wet weight of the distal $6 \mathrm{~cm}$ was recorded and used as an index of disease-related intestinal wall thickening. When the colons were shorter than $6 \mathrm{~cm}$, the entire colon was removed and weight was recalculated for $6 \mathrm{~cm}$. Subsequently, the colons were divided longitudinally into two parts and both were rolled up. For all animals, one role was used for histological analysis. In five randomly selected animals from each group, the other role was used for cytokine detection by means of a cytometric bead array (CBA) and for immunofluorescence of Zonula Occludens protein 1 (ZO-1) in the other five mice from each group. Besides the colon, for all animals, spleens were also sampled, weighed, and used for cytokine detection. All sampled tissues were frozen in liquid nitrogen and stored at $-80^{\circ} \mathrm{C}$ until analysis.

\section{Histological Analysis}

The longitudinally divided rolled-up parts of the colon, which were used for routine histology, were directly fixed in $4 \%$ formaldehyde and embedded in paraffin. Three transverse slices $(5 \mu \mathrm{m})$, taken from each colonic sample, were stained with hematoxylin-eosin and examined by light microscopy. Colonic inflammation was evaluated in a blind manner by estimating the 1) percentage of involved area, 2) the amount of follicles, 3) edema, 4) erosion/ulceration, 5) crypt loss, 6) infiltration of polymorphonuclear cells, and 7) infiltration of mononuclear cells. The percentage of area involved, erosion/ulceration, and the crypt loss was scored on a scale ranging from 0 to 4 as follows: 0 -normal, $1-$ less than 10\%, 2-10-25\%, $3-25-50 \%$, and $4-$ more than $50 \%$. Follicle aggregates were counted and scored as follows: 0-zero to one follicles, 1-two to three follicles, 2-four to five follicles, and 3-six follicles or more. The severity of the other parameters was scored on a scale from 0 to 3 as follows: 0-absent, 1-weak, 2-moderate, and 3-severe. All scores on the individual parameters together could results in a total score ranging from 0 to 24 .

\section{Immunofluorescence of Zonula Occludens Protein-1 (ZO-1)}

To examine the distribution of tight junctions, we stained three colon sections of five mice per group for ZO-1. Frozen sections of rolled-up colons were cut $(6 \mu \mathrm{m})$, allowed to air dry, fixed with $4 \%$ paraformaldehyde, and then washed three times with cold PBS. The sections were blocked with 1:10 normal goat serum and then incubated for $1 \mathrm{~h}$ at room temperature with rabbit anti-ZO-1 polyclonal antibody (Zymed, San Francisco, CA). As a negative control, sections were incubated with PBS- $0.1 \%$ BSA. Thereafter, sections were washed three times with cold PBS, incubated with Texas Red conjugated goat antirabbit antibody (Jackson, West Grove, $\mathrm{PA}$ ) for $50 \mathrm{~min}$ at room temperature and washed three times with PBS. Next, sections were incubated with $4^{\prime}, 6-$ diamino-2-phenyl indole (DAPI) $(0.5 \mathrm{mg} / \mathrm{mL}$, Sigma, St. 
Louis, MO) for 2 min, washed three times with PBS, dehydrated in ascending ethanol series, and mounted in mounting solution (Dako Cytomation, Ely, UK). Distribution of tight junctions was recorded with Metasystems Image Pro System (Metasystems, Sandhausen, Germany) mounted on a Leica DM-RE fluorescence microscope (Leica, Wetzler, Germany). Photomicrographs were recorded at a $200 \times$ magnification.

\section{Homogenisation and Cytometric Bead Array (CBA)}

For analysis of cytokine concentrations, homogenates were made from colon and spleen samples with a tissue homogenizer in 9 volumes (w/v) Greenberger lysis buffer $(300 \mathrm{mmol} / \mathrm{L} \mathrm{NaCl}, 15 \mathrm{mmol} / \mathrm{L}$ Tris, $2 \mathrm{mmol} / \mathrm{L}$ $\mathrm{MgCl}_{2}, 2 \mathrm{mmol} / \mathrm{L}$ Triton X-100 (Sigma, St. Louis, MO), Pepstatin A, Leupeptin, Aprotinine (Roche, Mannheim, Germany), all $20 \mathrm{ng} / \mathrm{mL}$; $\mathrm{pH}$ 7.4). The tissues were lysed in the Greenberger lysis buffer for $1 \mathrm{~h}$ on ice and centrifuged for $7 \mathrm{~min}$ at $3000 \mathrm{rpm}$ and for $10 \mathrm{~min}$ at $14,000 \mathrm{rpm}$. The supernatant was collected and stored at $-80^{\circ} \mathrm{C}$ until cytokine analysis using a cytometric bead array. The mouse inflammation and Th1/Th2 CBA (Becton Dickinson Biosciences, San Diego, CA) were used for colon homogenates and the mouse inflammation CBA in spleen homogenates to determine simultaneously the concentrations of various cytokines in colon (interleukin (IL)-2, IL-4, IL-6, IL-10, IL-12p70, monocyte chemotactic protein (MCP)-1, interferon (IFN)- $\gamma$ and tumor necrosis factor (TNF)- $\alpha$ ) and spleen (IL-6, IL-10, IL-12p70, MCP-1 and TNF- $\alpha$ ) homogenates according to the instructions of the manufacturer. Briefly, $5 \mu \mathrm{L}$ of sample or the cytokine standard mixture was mixed with $5 \mu \mathrm{L}$ of the mixed capture beads and $5 \mu \mathrm{L}$ of the detection antibodyphycoerythrin (PE) reagent and incubated at room temperature for $2 \mathrm{~h}$ in the dark. Two-color flow cytometric analysis was performed using a FACScan ${ }^{\circledR}$ flow cytometer (Becton Dickinson Immunocytometry Systems (BDIS), San Jose, CA). Data were acquired and analyzed using Becton Dickinson CBA software.

\section{Myeloperoxidase (MPO) in Colon Homogenates and Plasma}

To quantify the extent of colonic neutrophil accumulation and systemic neutrophil amounts, MPO concentrations in colon homogenates and EDTA plasma were analyzed by enzyme-linked immunosorbent assay (ELISA) (HBT, Uden, The Netherlands). In brief, 96 well plates (Greiner Bio-one, Frickenhausen, Germany) were coated with $1.5 \mu \mathrm{g} / \mathrm{mL}$ monoclonal MPO antibodies. Immobilized MPO was detected using a specific biotinylated rabbit-antihuman MPO antibody, followed by the addition of peroxidase-conjugated streptavidin and tetramethylbenzidine (TMB) substrate. All samples of both groups were analyzed on one plate. The detection limit of the MPO ELISA was $1.02 \mathrm{ng} / \mathrm{mL}$. The intraassay variation was less than $7.5 \%$.

\section{Serum Amyloid P Component (SAP) in Plasma}

To evaluate the acute phase reaction activity, SAP concentrations were analyzed in EDTA plasma by ELISA, as described in (14). Briefly, 96 well plates (Greiner Bioone, Frickenhausen, Germany) were coated with $3 \mu \mathrm{g} / \mathrm{mL}$ sheep-antimouse SAP (Calbiochem, San Diego, CA) antibodies. Immobilized SAP was detected using a specific biotinylated rabbit-antimouse SAP antibody (Calbiochem), followed by the addition of peroxidase-conjugated streptavidin (Zymed Laboratories, San Francisco, CA) and TMB substrate (Kirkegaard \& Perry Laboratories, Gaithersburg, MD). All samples of both groups were analyzed on one plate. The detection limit of the ELISA was $5 \mathrm{ng} / \mathrm{mL}$. The intraassay variation was less than $5 \%$.

\section{Statistical Analysis}

All data, except body weights (means \pm SEM), are expressed as dot plots in which the median is indicated. Differences between the groups were analyzed using the Mann-Whitney $U$ test. Two analysis of covariance (ANCOVA) models were used to analyze changes in body weight. The first one evaluated the effects of rosiglitazone on body weight at the end of 16 days rosiglitazone treatment using weight at the start of rosiglitazone treatment (day 0) as covariate and the second model evaluated the effects of rosiglitazone on body weight at the end of 7 days DSS treatment (day 23) using weight at the start of DSS treatment (day 16) as covariate. Correlation analyses were performed using the Spearman correlation test. Values of $P<0.05$ were considered statistically significant. SPSS 10 for Macintosh (SPSS, Chicago, IL) was used for the analysis.

\section{RESULTS}

\section{Effects of Rosiglitazone on Colitis Severity}

During the 16 days, preceding colitis induction, mice treated with rosiglitazone gained more weight $(15.3 \pm 2.7 \%)$ than control mice $(5.6 \pm 2.1 \%)(P<0.001)$ (Fig. 1A). After 7 days DSS, the rosiglitazone-treated mice had lost significantly more weight $(-13.5 \pm 4.0 \%$ of their initial weight at DSS induction) than the control mice $(-3.8 \pm 5.0 \%)(P<0.001)$ (Fig. 1B). 
A
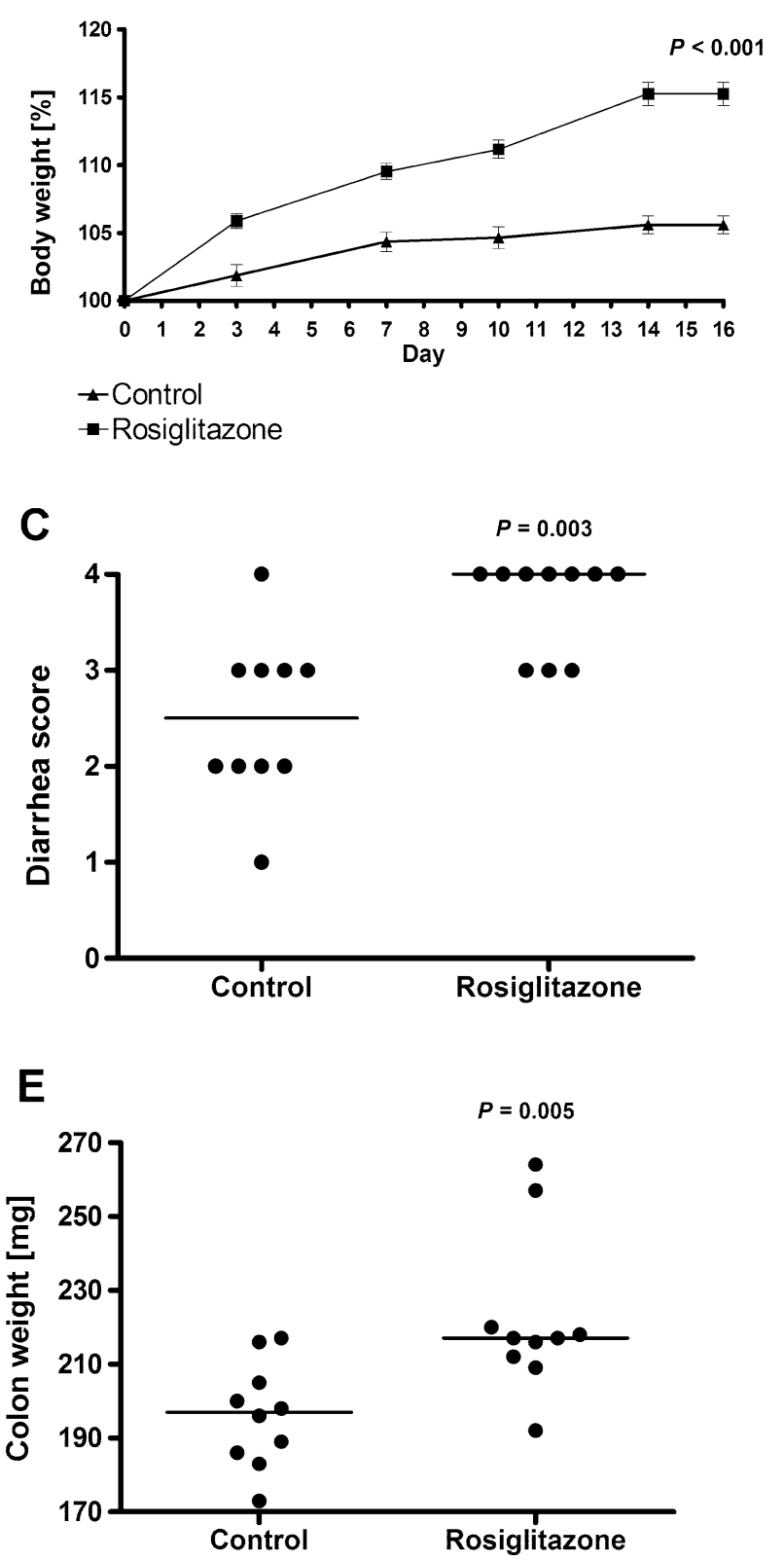

B

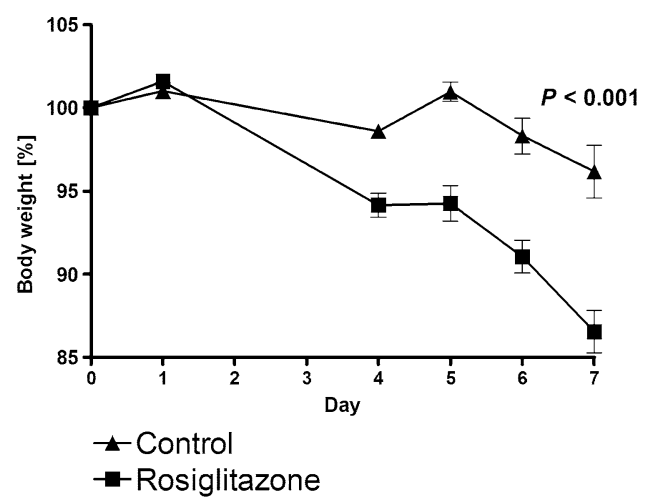

D

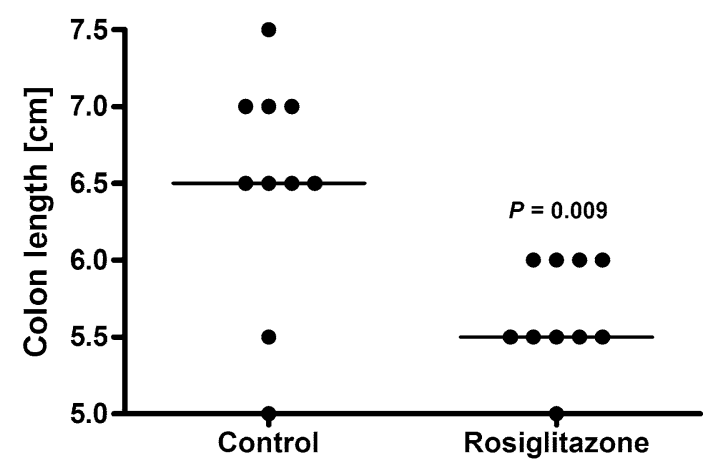

$\mathbf{F}$

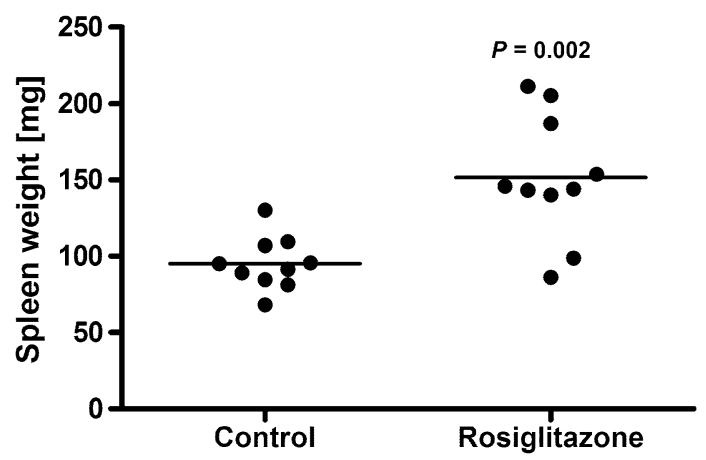

Fig. 1. Treatment with rosiglitazone increased DSS colitis disease severity. Ten female mice consumed a control diet or the same diet with $12 \mathrm{mg} / 100 \mathrm{~g}$ rosiglitazone for 16 days prior to DSS colitis induction. Colitis was induced by $1.5 \%$ (w/v) DSS in the drinking water for 7 days. Body weight was recorded prior to colitis induction (A) and after colitis induction as a measure of disease activity (B). Disease severity was measured 7 days after DSS colitis induction by a diarrhea score (C), colon length (D), colon weight of last $6 \mathrm{~cm}$ (E) and spleen weight $(\mathrm{F})$.

Seven days after DSS-colitis induction, diarrhea of the mice was scored (Fig. 1C). In agreement with the more pronounced loss of body weight, the rosiglitazone-treated mice had more diarrhea (median score 4.0) than control mice (score 2.5) $(P=0.003)$.
After sacrificing the mice, the total length of the colon and the weight of the last $6 \mathrm{~cm}$ of the colon were determined and used as indicators of disease-related colon shortening and intestinal wall-thickening. The weight of the colon $(217 \mathrm{mg})$ was significantly increased and the 

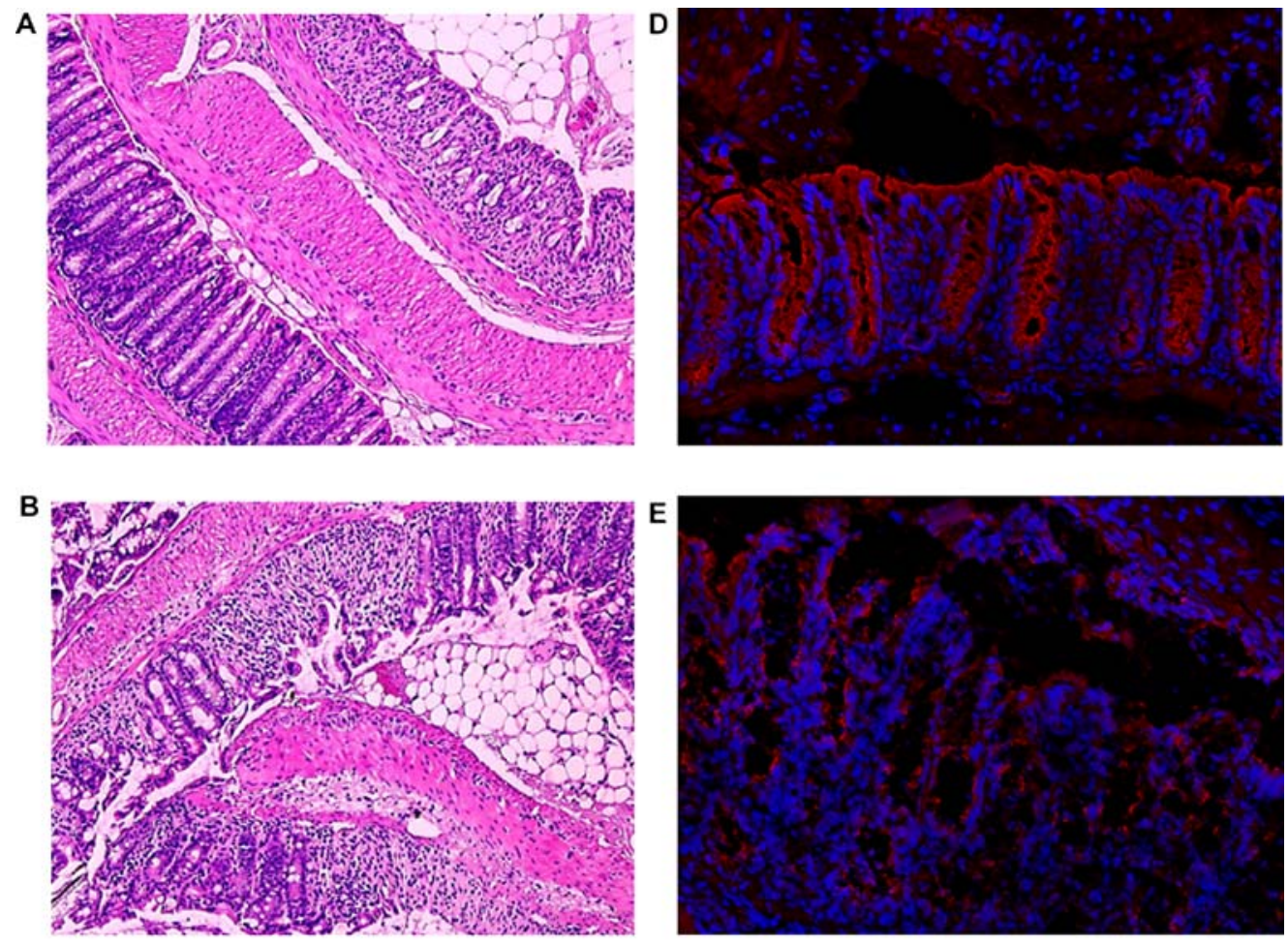

C

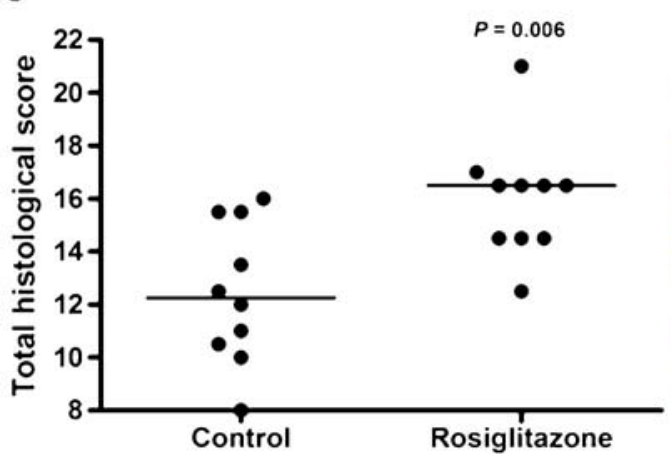

$\mathbf{F}$

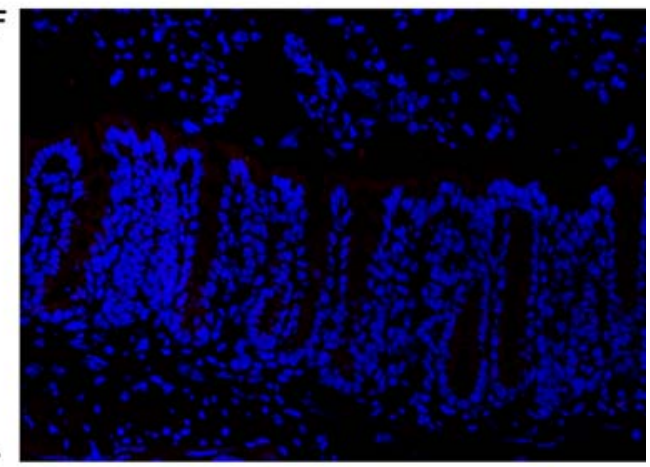

Fig. 2. Treatment with rosiglitazone increased histological score and induced a loss of tight junction protein ZO-1. Ten female mice consumed a control diet or the same diet with $12 \mathrm{mg} / 100 \mathrm{~g}$ rosiglitazone for 16 days prior to DSS colitis induction. Colitis was induced by $1.5 \%$ (w/v) DSS in the drinking water for 7 days. (A-C) Histological examination was scored in haematoxylin-eosin (HE) stained sections of colons at 7 days after DSS colitis induction (magnification $25 \times$ ). (A) HE staining of representative section of a control mouse and (B) rosiglitazone-treated mouse. (C) Total histological score of control and rosiglitazone-treated mice. (D-F) Immunolocalization of ZO-1 (red) showed a relative regular distribution in the colon of control colitis mice (D). ZO-1 is localized in the upper part of the enterocytes, showing a normal distribution in association with the cellular surface. Although parts of the colon of control colitis mice showed ZO-1 loss, rosiglitazone treatment (E) led to significant more loss of ZO-1. Moreover, the colon tissue was more disrupted and disorganized, illustrated by an irregular distribution of nuclei (blue). Although tissue sections of rosiglitazone-treated mice showed parts with a normal distribution of ZO-1 the difference with the control mice was striking. (F) Negative control staining of a colon section of a control mouse. The histology shown is representative for all tissue sections studied (magnification $200 \times$ ). 
Table I. Different Items of Histology Scores of the Colon of Rosiglitazone-Treated and Control DSS Colitis Mice

\begin{tabular}{lcc}
\hline & $\begin{array}{c}\text { Rosiglitazone } \\
(n=10)\end{array}$ & $\begin{array}{c}\text { Control } \\
(n=10)\end{array}$ \\
\hline Total score & $16.5^{*}(12.5-21.0)$ & $12.3(8.0-16.0)$ \\
Area involved $^{a}$ & $4.0(4.0-4.0)$ & $4.0(3.0-4.0)$ \\
Follicle aggregates $^{b}$ & $1.0(0.0-3.0)$ & $0.0(0.0-2.0)$ \\
Edema $^{b}$ & $2.5^{*}(1.0-3.0)$ & $1.8(1.0-2.0)$ \\
Ulceration $^{a}$ & $2.0^{*}(2.0-3.0)$ & $1.0(1.0-3.0)$ \\
Crypt loss $^{a}$ & $3.0^{*}(2.0-3.0)$ & $2.0(1.0-3.0)$ \\
Polymorphonuclear cells $^{b}$ & $2.0(1.5-3.0)$ & $1.8(0.5-2.5)$ \\
Mononuclear cells $^{b}$ & $1.5(1.0-2.0)$ & $1.5(1.0-2.0)$ \\
\hline
\end{tabular}

${ }^{a}$ Median scores (minimum-maximum) on a scale of 0-4;0 $=$ normal, $1=$ less than $10 \%, 2=10-25 \%, 3=25-50 \%$, and $4=$ more than $50 \%$.

${ }^{b}$ Median scores (minimum-maximum) on a scale of $0-3 ; 0=$ absent, $1=$ weak, $2=$ moderate, $3=$ severe.

${ }^{*} P<0.05$ versus control group.

length $(5.5 \mathrm{~cm})$ of the colon was significantly decreased in the rosiglitazone group $(P=0.005$ and $P=0.009$, respectively) as compared to control group (197 $\mathrm{mg}$ and $6.5 \mathrm{~cm}$, respectively) (Fig. 1D-E). Also, the weight of the spleen was significantly increased in the rosiglitazonetreated mice $(145 \vee 93 \mathrm{mg})(P=0.002)$ (Fig. 1F). Colon weight correlated positively with spleen weight $(r=0.466, P=0.038)$ and diarrhea score $(r=0.509$, $P=0.022$ ).

Colonic inflammation was also evaluated by histological analysis. Figures 2A-B show representative pictures of the colons from control and rosiglitazone-treated mice. Colonic inflammation in the control mice was mostly continuous (most severe at the anal side), whereas the colonic inflammation of the rosiglitazone-treated mice was characterized by skipped-lesions (isolated affected areas with intervening quite normal mucosa). The total colitis score was significantly increased in the rosiglita- zone group (16.5 points) as compared to the control group (12.3 points; $P=0.006$ ) (Fig. 2 C), which was mainly caused by an increase in ulceration $(P=0.011)$, crypt loss $(P=0.007)$ and edema $(P=0.043)$ (Table $\mathrm{I})$. Total histological score correlated positively with colon weight $(r=0.514, P=0.020)$.

Immunofluorescence showed that ZO-1 appearance in colons of rosiglitazone-treated mice was less distinct, diffusely localized, and even a loss of ZO-1 protein was observed in several parts of the tissue sections as compared to control mice (Fig. 2D-F).

\section{Effects of Rosiglitazone on Colon and Spleen Proinflam- matory Cytokine Concentrations}

The concentration of different cytokines in colon and spleen homogenates were analyzed by a CBA to evaluate the extent of the local immune responses. The colonic concentrations of TNF- $\alpha(P=0.028)$ and IFN- $\gamma(P=0.009)$ were decreased in the rosiglitazone group (Table II). IL-2, IL-4, IL-6, IL-10, IL-12p70, and MCP-1 concentrations in the colon homogenates were not significantly different between the two groups. IL-12p70 concentrations in colon homogenates correlated with TNF- $\alpha$ and IFN- $\gamma$ (both $r=0.664, P=0.044$ ) and TNF- $\alpha$ also correlated with IFN- $\gamma(r=0.891$, $P=0.001)$. Furthermore, MCP-1 correlated with IL-6 $(r=0.685, P=0.029)$. Not only in the colon, but also in the spleen, TNF- $\alpha$ concentrations were significantly $(P=0.028)$ decreased. However, spleen IL-10 concentrations were significantly $(P=0.013)$ increased in the rosiglitazone-treated mice (Table II). IL-6, IL-12p70, and MCP-1 concentrations in spleen were not different between the two groups.

Table II. Cytokine Concentrations in Colon and Spleen Homogenates

\begin{tabular}{lllll}
\hline & \multicolumn{2}{c}{ Colon } & & \multicolumn{2}{c}{ Spleen } \\
\cline { 2 - 3 } Cytokine $^{a}$ & \multicolumn{1}{c}{$\begin{array}{c}\text { Rosiglitazone } \\
(n=5)\end{array}$} & Control $(n=5)$ & $\begin{array}{c}\text { Rosiglitazone } \\
(n=10)\end{array}$ & $\begin{array}{c}\text { Control } \\
(n=10)\end{array}$ \\
\hline IL-12p70 & $8.6^{b}(6.6-17.9)$ & $10.0(9.1-17.2)$ & $4.1(0.0-5.7)$ & $1.4(0.0-6.7)$ \\
TNF- $\alpha$ & $48^{*}(38-83)$ & $84(41-229)$ & $57^{*}(30-106)$ & $82(54-106)$ \\
IFN- $\gamma$ & $1.9^{*}(1.6-3.0)$ & $8.0(3.4-37.2)$ & ND & ND \\
MCP-1 & $867(455-1131)$ & $477(391-950)$ & $3.3^{*}(54-191)$ & $152(101-342)$ \\
IL-10 & $8.4(0.0-22.4)$ & $9.6(7.5-17.8)$ & $25(16-33)$ & $0.0(0.0-0.0)$ \\
IL-6 & $390(96-1327)$ & $272(151-1249)$ & ND & $36(13-290)$ \\
IL-4 & $0.0(0.0-3.9)$ & $0.0(0.0-5.0)$ & ND & ND \\
IL-2 & $2.6(2.0-3.4)$ & $2.8(2.1-6.3)$ & ND \\
\hline
\end{tabular}

Note. ND not determined.

${ }^{a}$ Concentrations in $\mathrm{pg} / \mathrm{mL}$.

${ }^{b}$ Median (minimum-maximum).

${ }^{*} P<0.05$ versus control group. 
Effects of Rosiglitazone on MPO and SAP Concentrations in Colon Homogenates and/or Plasma

To determine colonic neutrophil accumulation and systemic neutrophil levels, MPO concentrations were analyzed in colon homogenates and plasma. No significant differences between the groups were found, neither in colon homogenates nor in plasma $(P=0.754$ and $P=0.174$, respectively) (Fig. 3A-B). MPO concentrations in colon homogenates correlated positively with MCP-1 concentrations in colon homogenates $(r=0.673$, $P=0.033)$.

To determine the extent of the acute phase response, we analyzed plasma SAP concentrations. The SAP concentration of the plasma of rosiglitazone-treated mice was significantly increased compared to control mice $(885 \mathrm{ng} / \mathrm{mL}$ versus $196 \mathrm{ng} / \mathrm{mL})(P=0.041)$ (Fig. 3C). SAP concentration in plasma correlated positively with total histological score $(r=0.540, P=0.014)$ and diarrhea score $(r=0.619, P=0.004)$.

\section{DISCUSSION}

The present study showed that in mice, 16 days pretreatment with rosiglitazone in the diet increased colonic inflammation after experimentally DSS-induced colitis. In contrast with all earlier studies, this is the first study that showed adverse effects of a PPAR $\gamma$ agonist in a colitis model. Earlier studies administrated various PPAR agonists (troglitazone, pioglitazone, rosiglitazone, netoglitazone) by oral gavage on the first day of colitis induction $(4,12), 1$ or 2 days before colitis induction $(4,5,8,10)$, or after colitis induction $(5,6,12)$ until sacrificing the mice. Each of these studies showed decreased disease activity, less mortality, and less colonic (Th1) cytokine production $(4-6,8,10,12)$. Also, intraperitoneal injection of pioglitazone - starting at the day of DSS colitis induction until day 7-showed preventive effects (11). Moreover, rosiglitazone in the diet, 2 days before colitis induction protected against acute DSS-colitis (7). The most striking difference in the design of all these studies as compared to ours is the time at which PPAR $\gamma$ agonist treatment was initiated. In our experimental model, we have deliberately chosen to test the effects of a PPAR $\gamma$ agonist in a longer term preventive manner to lower the risk of relapses. We therefore applied rosiglitazone for 16 days prior to DSS-induced colitis induction. Only in one other study, rosiglitazone was added to the diet for a longer period (9). In that study, Lytle et al. examined the effects of rosiglitazone treatment in the diet for a period of 12 weeks on the spontaneous development of colitis in IL-10
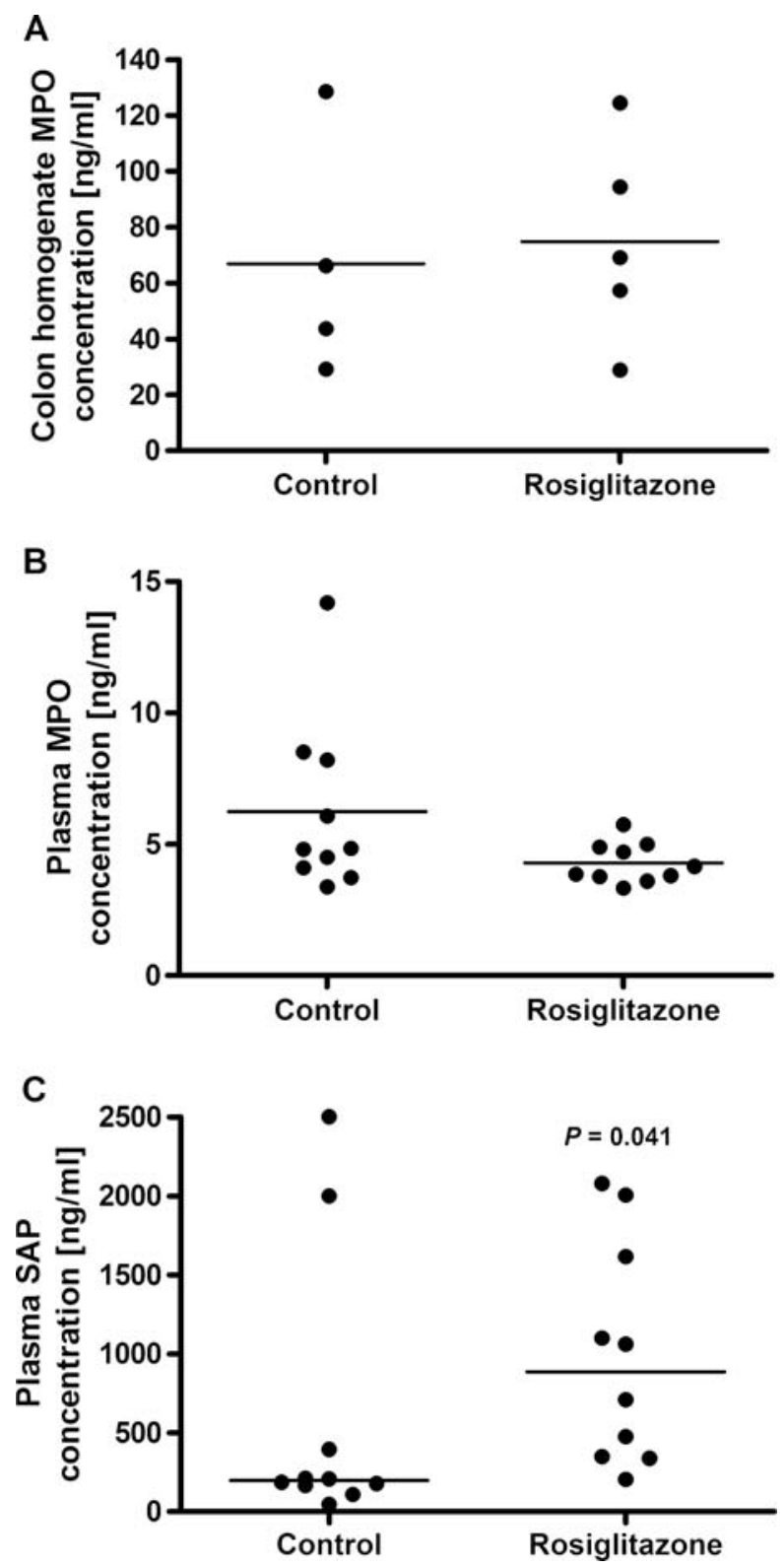

Fig. 3. Treatment with rosiglitazone did not change MPO concentrations in colon homogenates and plasma, but increased SAP concentrations in plasma. Ten females mice consumed a control diet or the same diet with $12 \mathrm{mg} / 100 \mathrm{~g}$ rosiglitazone for 16 days prior to DSS colitis induction. Colitis was induced by $1.5 \%(\mathrm{w} / \mathrm{v})$ DSS in the drinking water for 7 days. MPO concentrations were determined in colon homogenates (A) and in plasma (B) and SAP concentrations were determined in plasma (C) 7 days after colitis induction.

- / - mice. In contrast to our study, this study showed that longer term treatment with rosiglitazone slowed down the onset of spontaneous severe colitis compared to a control diet. However, rosiglitazone did not prevent colitis development and in the end, disease severity was comparable 
between rosiglitazone-treated and control animals. However, in contrast to our study, rosiglitazone did not worsen the disease state. It should be realized, however, that the IL10 $-/-$ model is a different model as compared to the DSS model. Whereas the IL-10 - / - model depends on a disregulated immunological response, which results in spontaneous colitis development, the DSS model is related to a disruption of epithelial cell barrier function, which results in acute colitis.

In all studies in which rosiglitazone was administrated as a gavage, daily intakes varied between $1-50 \mathrm{mg} / \mathrm{kg}$. Optimal (preventive and therapeutic) effects were found at $20 \mathrm{mg} / \mathrm{kg}$, while higher doses had no additional beneficial effects $(4-6,12)$. In the two studies in which rosiglitazone was mixed with powdered chow and used in a preventive mode, animals consumed daily about $20 \mathrm{mg} / \mathrm{kg}$ (7, 9). It can be calculated that in our study, daily intake was comparable. Therefore, differences in effects on colitis cannot be explained by differences in rosiglitazone dosage. However, in future studies, a dose response relationship for the longer term preventive (dietary) mode should be evaluated. Another possibility could be differences in susceptibility between mice of various genetic backgrounds. We used C57BL/6 mice whereas other DSS studies used not only C57BL/6 $(5,8)$, but also balb/c (10, 11) and Swiss-Webster mice (12). However, these DSS studies using gavages did not show any differences in effects between strains. A possible explanation therefore is that-in contrast to the earlier studies-longer term rosiglitazone treatment before colitis induction induced changes in the intestinal barrier, which made the intestine more susceptible to intestinal damage. In the DSS model, which depends on intestinal barrier disruption, this increased susceptibility may induce more severe intestinal damage. This hypothesis however needs to be tested in future studies, for example, by comparing acute curative effects of rosiglitazone versus long-term preventive effects side-by-side in one experiment and by confirming this data in another model of IBD.

Changes in weight loss, diarrhea, colon weight and length, spleen weight, histological scores, and plasma SAP concentration indicated that colitis was more severe in rosiglitazone-treated mice. Although the mice gained more weight before colitis induction, they also lost more weight after 7 days DSS colitis. Weight gain is a known adverse effect of rosiglitazone treatment in humans $(15,16)$ and in mice $(17)$, which is also mentioned in the instructions of the Avandia tablets. Moreover, also other parameters-diarrhea, colon weight and length, spleen weight, histological scores, and plasma SAP concentration-indicate that colitis was more severe in rosiglitazone-treated mice. However, TNF- $\alpha$ lev- els in colon and spleen and IFN- $\gamma$ levels in colon were decreased, and those of IL-10 (an antiinflammatory cytokine) in the spleen were increased in the rosiglitazonetreated mice. Moreover, other cytokines (IL-2, IL-4, IL6, IL-12p70, and MCP-1) and MPO concentrations in colon and/or spleen homogenates and/or plasma showed no differences. Why these inflammatory proteins did not show an increase as expected from disease severity is not known. At first, the combination of results seems contradictive, i.e., lower expression of proinflammatory cytokines in the colon, no change in colonic MPO concentrations, and a more severe DSS-induced colitis phenotype as evaluated by - among others-weight loss and histological scores. There are, however, different possibilities explaining the simultaneous appearance of these characteristics. One possible explanation is that mice were too sick to produce cytokines at high concentrations, as has also been found in another study (18). This would imply that the acute innate inflammatory response during the first days of DSS treatment in the rosiglitazone animals was so strong that at day 7 , when the mice were sacrificed, the local inflammatory cells were exhausted. However, the increased IL-10 concentration does not support this explanation and suggests an enhanced antiinflammatory immune response. Therefore, a more likely explanation is that the absence of elevated cytokine concentrations is caused by the down-regulation of the proinflammatory response of intestinal and immune cells by rosiglitazone. Other studies with PPAR $\gamma$ agonists also showed decreased colonic Th1 cytokines $(4,5,10,12)$ and an increase in colonic IL-10 mRNA (5). However, in contrast to our study, in these earlier studies, colitis was attenuated. We now suggest that the down-regulated immune response due to long-term rosiglitazone pretreatment will ultimately result in a situation where there is no adequate immune response toward the injured epithelium by DSS. This will enhance the possibility of bacterial translocation, consequently leading to more pronounced disease severity. Also, differences in the distribution of the colonic damage (continuous from anal toward the small intestine in control animals and skipped-lesions in rosiglitazone animals) suggest a different type of colitis/colonic injury due to rosiglitazone interference. In relation to this latter explanation, it is more likely that rosiglitazone pretreatment makes the intestinal wall more vulnerable for DSSinduced tissue injury. Although in a future study we should measure tight junction distribution in rosiglitazone-treated mice before colitis induction, the striking loss of ZO1 protein in rosiglitazone-treated colitis mice as compared to control colitis mice seems suggestive. We do realize that this does not provide any evidence for the assumption that 16 days rosiglitazone pretreatment makes 
the intestinal wall more susceptible for the DSS-induced tissue injury; however, with respect to the severe outcome, we did not want to postpone this knowledge for too long. Moreover, the increase in spleen weight in the rosiglitazone-treated animals is of potential concern. It could simply be a result of the more severe colitis in the rosiglitazone-treated animals. At this moment, however, we cannot exclude that it is the result of rosiglitazone treatment per se.

In conclusion, our results indicate that rosiglitazone when added to the diet in a preventive approach for a longer period (16 days) results in a higher susceptibility for DSS-induced colitis. This finding is in sharp contrast to promising results of earlier studies in which PPAR $\gamma$ agonists were given shortly (i.e., 1 or 2 days) before experimental colitis induction and longer term dietary treatment in a spontaneous IL-10 - / - colitis model. We hypothesize that rosiglitazone pretreatment makes the intestinal wall more susceptible for DSS-induced tissue injury ultimately resulting in a more pronounced disease severity. The phenotypic characteristics suggest that the PPAR $\gamma$ activation keeps the inflammatory response dampened despite the higher tissue injury by DSS. Future research is warranted to unravel the mechanism underlying these unexpected effects and confirm this data in other IBD models.

\section{ACKNOWLEDGMENTS}

We thank Joost Daalhuisen for his excellent biotechnical assistance and Prof. Dr. Fiebo J. ten Kate for microscopic scoring of the paraffin sections. We thank Joep Derikx and Mohammed Hadfoune for assistance with MPO and SAP measurements.

\section{REFERENCES}

1. Delerive P, Fruchart JC, Staels B: Peroxisome proliferator-activated receptors in inflammation control. J Endocrinol 169:453-459, 2001

2. Wang Q, Herrera-Ruiz D, Mathis AS, Cook TJ, Bhardwaj RK, Knipp GT: Expression of PPAR, RXR isoforms and fatty acid transporting proteins in the rat and human gastrointestinal tracts. J Pharm Sci 94:363-372, 2005

3. Auboeuf D, Rieusset J, Fajas L, Vallier P, Frering V, Riou JP, et al:: Tissue distribution and quantification of the expression of mRNAs of peroxisome proliferator-activated receptors and liver $\mathrm{X}$ receptor-alpha in humans: No alteration in adipose tissue of obese and NIDDM patients. Diabetes 46:1319-1327, 1997

4. Desreumaux P, Dubuquoy L, Nutten S, Peuchmaur M, Englaro $\mathrm{W}$, Schoonjans $\mathrm{K}$, et al:: Attenuation of colon inflammation through activators of the retinoid $\mathrm{X}$ receptor $(\mathrm{RXR})$ /peroxisome proliferator-activated receptor gamma (PPARgamma) heterodimer. A basis for new therapeutic strategies. J Exp Med 193:827-838, 2001

5. Saubermann LJ, Nakajima A, Wada K, Zhao S, Terauchi Y, Kadowaki T, et al.: Peroxisome proliferator-activated receptor gamma agonist ligands stimulate a Th2 cytokine response and prevent acute colitis. Inflamm Bowel Dis 8:330-339, 2002

6. Sanchez-Hidalgo M, Martin AR, Villegas I, Alarcon De La, Lastra $\mathrm{C}$ : Rosiglitazone, an agonist of peroxisome proliferator-activated receptor gamma, reduces chronic colonic inflammation in rats. Biochem Pharmacol 69:1733-1744, 2005

7. Adachi M, Kurotani R, Morimura K, Shah Y, Sanford M, Madison $\mathrm{BB}$, et al.: Peroxisome proliferator activated receptor gamma in colonic epithelial cells protects against experimental inflammatory bowel disease. Gut 55:1104-1113, 2006

8. Schaefer KL, Denevich S, Ma C, Cooley SR, Nakajima A, Wada K, et al.: Intestinal antiinflammatory effects of thiazolidenedione peroxisome proliferator-activated receptor-gamma ligands on $\mathrm{T}$ helper type 1 chemokine regulation include nontranscriptional control mechanisms. Inflamm Bowel Dis 11:244-252, 2005

9. Lytle C, Tod TJ, Vo KT, Lee JW, Atkinson RD, Straus DS: The peroxisome proliferator-activated receptor gamma ligand rosiglitazone delays the onset of inflammatory bowel disease in mice with interleukin 10 deficiency. Inflamm Bowel Dis 11:231-243, 2005

10. Takaki K, Mitsuyama K, Tsuruta O, Toyonaga A, Sata M: Attenuation of experimental colonic injury by thiazolidinedione agents. Inflamm Res 55:10-15, 2006

11. Takagi T, Naito Y, Tomatsuri N, Handa O, Ichikawa H, Yoshida $\mathrm{N}$, et al:: Pioglitazone, a PPAR-gamma ligand, provides protection from dextran sulfate sodium-induced colitis in mice in association with inhibition of the NF-kappaB-cytokine cascade. Redox Rep 7:283-289, 2002

12. Su CG, Wen X, Bailey ST, Jiang W, Rangwala SM, Keilbaugh SA, et al:: A novel therapy for colitis utilizing PPAR-gamma ligands to inhibit the epithelial inflammatory response. J Clin Invest 104:383389, 1999

13. Lewis JD, Lichtenstein GR, Stein RB, Deren JJ, Judge TA, Fogt $\mathrm{F}$, et al:: An open-label trial of the PPAR-gamma ligand rosiglitazone for active ulcerative colitis. Am J Gastroenterol 96:3323-3328, 2001

14. Daemen MA, Heemskerk VH, van't Veer C, Denecker G, Wolfs TG, Vandenabeele $\mathrm{P}$, et al:: Functional protection by acute phase proteins alpha(1)-acid glycoprotein and alpha(1)-antitrypsin against ischemia/reperfusion injury by preventing apoptosis and inflammation. Circulation 102:1420-1426, 2000

15. Lebovitz HE: Differentiating members of the thiazolidinedione class: a focus on safety. Diabetes Metab Res Rev 18(2):S23-29, 2002

16. Miyazaki Y, Glass L, Triplitt C, Matsuda M, Cusi K, Mahankali A, et al.: Effect of rosiglitazone on glucose and non-esterified fatty acid metabolism in Type II diabetic patients. Diabetologia 44:22102219, 2001

17. Muurling M, Mensink RP, Pijl H, Romijn JA, Havekes LM, Voshol PJ: Rosiglitazone improves muscle insulin sensitivity, irrespective of increased triglyceride content, in ob/ob mice. Metabolism 52:10781083,2003

18. Whiting CV, Bland PW, Tarlton JF: Dietary n-3 polyunsaturated fatty acids reduce disease and colonic proinflammatory cytokines in a mouse model of colitis. Inflamm Bowel Dis 11:340-349, 2005 